Ana Esther Escalante Ferrer; Luz Marina Ibarra Uribe; Leticia Pons Bonals;

Cuadernos de $\mathrm{H}$ Ideas, vol. 11, $\mathrm{n}^{\circ} .11$, e003, diciembre 2017

ISSN 2313-9048 | https://doi.org/10.24215/23139048e003

http://perio.un/p.edu.ar/ojs/index.php/cps/index

FPyCS | Universidad Nacional de La Plata

La Plata | Buenos Aires | Argentina

\title{
Docentes de dos universidades públicas mexicanas ante la integridad académica de sus estudiantes $\left(^{*}\right)$
}

\section{Teachers from two Mexican public universities before the academic integrity of their students}

\author{
Ana Esther Escalante Ferrer \\ Universidad Autónoma del Estado de Morelos, México. \\ anaescalante7@hotmail.com
}

\section{Luz Marina Ibarra Uribe}

Universidad Autónoma del Estado de Morelos, México.

marina.ibarra@uam.mx

\section{Leticia Pons Bonals}

Universidad Autónoma de Chiapas, México.

pbonals@hotmail.com

\section{Resumen}

El artículo contribuye a las reflexiones sobre la integridad académica en Instituciones de Educación Superior (IES). Se revisan experiencias de docentes de pregrado y posgrado de dos universidades públicas mexicanas, el propósito es conocer si las prácticas que atentan contra la integridad académica disminuyen conforme aumenta el grado de estudios y cuáles acciones implementan los docentes para promover la integridad.

Se aborda el concepto de integridad académica y se analiza cómo lo asumen las dos universidades estudiadas en su legislación. Posteriormente se describe la metodología y el análisis de las respuestas de 28 docentes a un cuestionario. Se encontraron sutiles diferencias en el nivel de integridad académica entre estudiantes de pregrado y posgrado y se mencionan las acciones implementas para fortalecerla. Se propone trascender el trabajo aislado de los docentes, así como la concertación de acciones que involucren a autoridades universitarias y a estudiantes para construir una ética institucional. 
Palabras clave: integridad académica; educación superior; pregrado; posgrado.

\section{Abstract}

The article contributes to reflections on academic integrity in Higher Education Institutions (HEls). We review experiences of undergraduate and graduate teachers from two Mexican public universities. The purpose is to know if practices that violate academic integrity decrease as the degree of studies increases and what actions teachers implement to promote integrity. It addresses the concept of academic integrity and analyzes how they (two universities studied) assume the integrity in theirs legislations. Subsequently, the methodology and analysis of the responses of 28 teachers to a questionnaire are described. Subtle differences were found in the level of academic integrity between undergraduate and graduate students and the actions implemented to strengthen the integrity are mentioned. It is proposed to transcend the isolated work of teachers, as well as the agreement of actions involving university authorities and students to build an institutional ethics.

Keywords: Academic integrity; higher education; undergraduate; postgraduate. 


\section{Introducción}

El propósito de este artículo es contribuir a las reflexiones que durante las últimas décadas han llevado a cuestionar la integridad académica en las universidades, esto es, ¿hasta dónde las universidades cumplen con los principios y valores en que sustentan su existencia y función social? Para ello, se presentan resultados de un estudio colectivo de casos, (1) llevado a cabo en dos universidades mexicanas: la Universidad Autónoma de Chiapas (UNACH) y la Universidad Autónoma del Estado de Morelos (UAEM), específicamente con 28 docentes, quienes en conjunto, imparten clases en seis posgrados inscritos en el Programa Nacional de Posgrados de Calidad (PNPC) del Consejo Nacional de Ciencia y Tecnología (CONACYT), cuyos propósitos se orientan a la formación de investigadores en el área de las ciencias sociales. El análisis realizado en cada caso tuvo como objetivo conocer si las prácticas que atentan contra la integridad académica disminuyen conforme aumenta el grado de estudios y cuáles acciones implementan los docentes para promover dicha integridad.

Atendiendo a los lineamientos del CONACYT, los docentes adscritos a los posgrados que tienen este reconocimiento de calidad, deben impartir al menos una asignatura al semestre en el nivel de pregrado, por lo que tienen la oportunidad de comparar el comportamiento académico de los estudiantes inscritos en ambos grados. Ante esto, la investigación inició con el supuesto de que las conductas que atentan contra la integridad académica son más comunes en estudiantes de pregrado que en los de posgrados orientados a la investigación. En estos últimos, las normas y sanciones impuestas son más estrictas dado que una parte importante de la formación alude a la búsqueda y uso honesto de la información requerida para la generación de conocimientos. Un segundo supuesto consideró que, ante una falta de normatividad, cada docente, de manera aislada, decide cuándo y cómo actuar para promover la integridad académica.

La estructura de este artículo contempla una primera parte en la que se aborda el concepto de integridad académica, precisando las maneras en las que es asumido en las dos universidades contempladas. Posteriormente se explica la metodología de investigación que hizo posible recabar la información analizada en los resultados, los cuales se presentan en la tercera parte, utilizando técnicas cuantitativas y cualitativas para someter a escrutinio los supuestos. En las conclusiones se destaca la necesidad de impulsar un trabajo colaborativo entre docentes y demás integrantes de la comunidad académica con la finalidad de orientar un actuar ético, ya que sólo de esta manera tendremos individuos e instituciones cada vez más íntegras.

\section{Integridad académica}


La integridad académica es definida por la Universidad de San Diego como "la adopción y adecuación de los valores fundamentales que deben regir toda actividad académica, siendo éstos: honestidad, confianza, responsabilidad, respeto y justicia o equidad". (2) Para efectos de este trabajo, la integridad consiste en asegurar que todos los integrantes de la comunidad universitaria se conduzcan de acuerdo con los principios y valores institucionales.

Para Bretag (3), la integridad académica abarca la honestidad, la confianza, el respeto, la imparcialidad y la responsabilidad, y su cumplimiento garantiza la credibilidad social de la educación en todos los niveles, incluida la investigación y sus resultados. En el ámbito de la formación de investigadores, la falta de integridad académica se asocia con aquello que es el sustrato mismo de su profesión: la generación de conocimientos útiles para la sociedad; tiene que ver "con la confiabilidad de los datos de la investigación -para los pares y para el público-, con la corrección de la literatura académica, con nociones de propiedad intelectual, de derechos morales, de originalidad en la ciencia, donde ésta última, incluso, está muy asociada con el concepto de plagio".(4)

Como tema de investigación, la integridad académica ha cobrado fuerza a partir de la década de 1980. En el artículo de Comas, (5) se hace un recuento de las investigaciones realizadas refiriendo los siguientes ejes temáticos:

"[...] comportamiento deshonesto del alumnado en el transcurso de pruebas escritas (Bowers, 1964; Davis, Pierce, Yandell \& Arnow, 1995; Diekhoff, LaBeff, Clark, Williams, Francis \& Haynes, 1996; Genereux \& McLeod, 1995; Haines, Diekhoff, LaBeff \& Clark, 1986; Rogers, 2006; Tibbetts, 1998; Whitley, 1998);

[...] el plagio, la falsificación de datos y resultados en trabajos, etc. (Ashworth, Bannister \& Thorne, 1997; Brimble et al., 2005; Joyce, 2007; Lin \& Wen, 2007; McCabe, 2001; Underwood \& Szabo, 2003)".

Comas et al. (6) refieren además trabajos pioneros en países hispanoamericanos. En España, el de Rey-Abella et al. Publicado en 2006, uno más, publicado en 2008 por Agnes, el cual fue auspiciado por la empresa francesa de desarrollo de software antiplagio Six Degrés. y el emprendido con alumnado de pregrado en la Universidad de Las Islas Baleares por Sureda et al. En Colombia, el de Mejías y Ordóñez de 2004, en Brasil el de Da Costa et al. de 2006 y el de Garcia-Barbastefano y Gomes-de-Souza de 2007. En Perú el de Saldaña et al., realizado en 2010.

En el contexto mexicano, en 2006 inició el Proyecto Interuniversitario de Ética Profesional precursor de la Red de Investigadores para el Estudio de la Integridad y Calidad Académica (RIEICA); en esta última participan 54 investigadores adscritos a ocho colectivos mexicanos y cuatro españoles de las siguientes universidades: Autónoma de Chiapas, Autónoma del Estado de Morelos, Autónoma de Guerrero, Autónoma de Tlaxcala, Autónoma de Yucatán, Nacional Autónoma de México y Veracruzana. Tres colectivos españoles estaban adscritos a la Universidad de Barcelona, Universidad de Las 
Islas Baleares, Universidad de Málaga y un colectivo interinstitucional agrupó a la Universidad Complutense de Madrid, Universidad Católica de Valencia y Universidad de Valencia. El proyecto inicial incluyó dos líneas de acción, la primera referente a la profesionalización del profesorado y la segunda a la integridad académica. (7)

En el caso de la segunda línea, la Red revisó y adaptó un cuestionario diseñado y aplicado en España por el grupo de Las Islas Baleares, (8) con el propósito de emprender estudios comparativos sobre las prácticas que cuestionan la integridad académica en las distintas universidades participantes y proponer algunas acciones en consecuencia. Uno de los productos es este artículo, así como otros tres que abordan el estudio de la deshonestidad académica en la UAEM y la Universidad Veracruzana;(9) en la Universidad Autónoma de Guerrero; (10) así como en la UNACH (11).

Las investigaciones realizadas evidencian que el interés por la integridad académica no se limita al campo de la investigación, sino que, en ocasiones influenciado por los resultados obtenidos, ha trascendido a las universidades como para ser atendido con acciones prácticas. Algunos ejemplos de esto se muestran a continuación.

En una conferencia organizada en 1992 por Donald McCabe de la Universidad de Rutgers, representantes de 31 Instituciones de Educación Superior (IES) discutieron los resultados alarmantes de una encuesta sobre integridad académica, ahí se concertó la creación del International Center for Academic Integrity, (12) con el objetivo de enfrentar la deshonestidad académica en la educación superior, promover la cultura de la integridad en las comunidades académicas y ofrecer servicios de consulta, difusión, evaluación, así como recursos para su defensa. Este organismo instituyó los siguientes seis valores: honestidad, confianza, equidad, respeto, responsabilidad y coraje, como guías del actuar académico. Desde entonces a la fecha se han incorporado a esta organización IES de otros países y se han celebrado encuentros internacionales anuales en los que se continúa trabajando con miras a fortalecer la integridad académica.

En México, en 2010, la Universidad de Monterrey se proclamó como la primera institución de América Latina miembro de este organismo internacional y propuso un Código de Honor que incluye valores acordes a los tiempos actuales: respeto, justicia, libertad, responsabilidad social, trabajo, dignidad, búsqueda permanente de la verdad, paz, pluralismo, sensibilidad a la expresión humana, trascendencia, servicio, fraternidad e integridad (13).

En la conferencia anual del International Center for Academic Integrity (ICAI), celebrada en Dubai en 2014, se compiló un documento con expresiones de estudiantes de pregrado, con las que llaman a robustecer la ética en la cultura académica, promoviendo conductas honestas, responsables, respetuosas, justas e íntegras, que busquen la verdad y que fortalezcan el coraje. A manera de ejemplo se recuperan a continuación tres de ellas: 
"Engañar académicamente es muy malo principalmente porque estás engañando a otros, el grado proporciona al estudiante una insignia de excelencia en su campo [...] $y$ si se obtiene con mentiras o trampas es un engaño [Yazan Al Kawadri] Si todos engañan mientras aprenden, no se aprenderá nada y no habrá progreso alguno en la vida [Zainab Al Hammadi] Las acciones no éticas pueden llevar al éxito momentáneo, pero las acciones éticas llevarán a un éxito de larga duración [Sami El Okaily]"(14).

Actualmente, los grados de avance que muestran las IES en materia de integridad académica son diversos; en algunas se trabaja en el cambio de normativas legales. Tal es el caso analizado por Ruipérez y García-Cabrero, (15) en instituciones alemanas que sancionan el plagio como "delito de estafa científica [...] con nuevos criterios más severos (Goec Kenjan, 2013; Linke, 2015). También se [... establecen] sanciones diferenciadas según la cantidad y calidad de los fragmentos plagiados [...y] se fomenta el uso de webs de referencia".

Estos autores explican que estas medidas están sirviendo de ejemplo en otros países como Austria en donde se fundó la Agencia Austriaca de la Integridad Científica y Gran Bretaña en donde se impulsó la conformación de la Red Europea de Oficinas de Integridad Científica, a la que se ha sumado la Agencia Estatal Consejo Superior de Investigaciones Científicas (CSIC) de España. (16)

Algunas universidades han emprendido acciones a favor de la integridad académica asumiendo, de manera explícita, la definición de los valores que orientan el actuar de sus comunidades, por ejemplo, la Universidad de Jaén contempla en su Biblioteca virtual un espacio denominado Abierto Buja con un portal específico dedicado al Plagio $y$ deshonestidad académica.(17) Entre los recursos que ofrece se encuentra un documento en el que asume como componentes de la integridad académica los siguientes valores: honestidad, confianza, justicia, respeto y responsabilidad, en el entendido que:

"La falta de honestidad interfiere con el libre intercambio de ideas. La falta de confianza atenta contra los procesos de enseñanza y aprendizaje. La falta de justicia mina la dimensión crítica de la búsqueda del conocimiento propia de la enseñanza. Sin respeto no es posible el diálogo público. Si no asumimos nuestra responsabilidad en el fomento y la defensa de estos valores no nos podemos constituir como una comunidad de aprendizaje íntegra y de altura. Estos cinco valores nos proveen razones y motivos para actuar". (18)

\section{Integridad académica en dos universidades mexicana la UNACH y la UAEM}

A diferencia de las experiencias señaladas, en la UNACH y la UAEM la integridad académica se aborda de manera indirecta, se puede rastrear en los principios o valores que quedan asentados en algunos de sus documentos oficiales, en los que se destaca la 
necesidad de dar cumplimiento a los fines que justifican su fundación, así como los principios o valores que deben guiar la consecución de sus fines (ver Tabla 1). Algunas consideraciones que hay que hacer respecto a los fines y valores expresados en los documentos de la UNACH y la UAEM son las siguientes:

a) No hay un único documento de carácter fundacional, Ilámese código de conducta ética o manual de buenas prácticas, en el que se reglamente el cumplimiento de la integridad académica. En las Leyes Orgánicas de ambas universidades se pone énfasis en las funciones (enseñanza, investigación, extensión), más no en los principios o valores que sustentarán su ejecución. Este vacío puede explicar, en parte, la presencia de conductas académicas deshonestas.

b) En 2010 la UAEM, como respuesta este vacío, incluye en el Modelo Universitario los principios y valores que orientan el quehacer de la universidad, y en su actual Plan Institucional de Desarrollo se retoman de él los "valores que constituirán la esencia del Código Ético Institucional', que actualmente está en proceso de consulta para su aprobación por parte de la comunidad universitaria.

c) La preocupación por hacer explícitos los valores académicos (aquellos que constituyen la integridad académica), se expresa en los documentos que se elaboran en los momentos de cambio de dirección administrativa (Proyecto Académico de la UNACH, Modelo Universitario y Plan de Desarrollo Institucional de la UAEM); lo que puede hacer depender su cumplimiento del periodo de esa administración. Si consideramos que son los valores de la integridad académica los que dan identidad y definen el sentido de la vida universitaria, se genera un grave problema, pues el cambio y redefinición de estos no permite su difusión permanente y asimilación como parte del actuar cotidiano de la comunidad académica.

d) Siguiendo lo expuesto en el inciso anterior, se da el caso que los valores se difuminan o subsumen a los intereses del momento, situación que se refleja con mayor claridad en el actual Proyecto Académico de la UNACH en el que dos atributos de la calidad educativa -relevancia y pertinencia- son asumidos, junto con la calidad, como valores académicos; de igual manera la atención equitativa que incluye la diversidad se despliega en dos valores; a estos se suman transparencia (a manera de rendición de cuentas), sustentabilidad y economía (uso eficiente de los recursos) como valores fundamentales. La UAEM, por su parte, interesada en la elaboración del Código Ético Institucional, ha definido siete valores (solidaridad, eticidad, compromiso, honestidad, libertad, justicia y responsabilidad social) 
haciendo depender de estos algunos atributos de conducta, con los que espera allanar el camino hacia la consecución de la integridad académica.

e) Es necesario que se abran caminos de diálogo y se generen debates que lleven a la definición de los principios y valores que la universidad considera necesarios para conducir un actuar académico íntegro, más allá de la idea de responder a la inmediatez. Poner sobre la mesa este asunto permitirá a las universidades resarcir la identidad institucional y responder a las críticas que actualmente reciben de parte de la sociedad. En el caso de la UNACH falta claridad, pues mientras en el actual Proyecto Académico se dejan de lado valores como: libertad, justicia, democracia, solidaridad e igualdad; estos se encuentran asentados como parte de las funciones universitarias que establece el Estatuto General, lo que refleja contradicciones entre ambos documentos e indefinición del ideario universitario; esta situación se agrava aún más cuando observamos que la honestidad ha quedado excluida

en

ambos documentos. 
TABLA 1. Fines y valores universitarios en la UNACH y la UAEM

Fines de la Universidad

Universidad Ley Orgánica de la UNACH: (19) Art. 2, Cap. II del Objeto y Facultades Estatuto General de la UNACH: (20) Art. 3, Cap. II. De los Objetivos

\section{En la Ley Orgánica:}

Impartir enseñanza superior para formar los profesionistas,

investigadores, profesores, universitarios y técnicos que requiere el desarrollo económico y social del estado; Il. Organizar, desarrollar y fomentar la investigación científica y humanística, considerando las condiciones y problemas nacionales y, primordialmente, los del Estado de Chiapas, y III. Extender, con la mayor amplitud posible, los beneficios de la cultura en todos los medios sociales de la comunidad que la

Universidad Autónoma de sustenta.

Chiapas (UNACH)

En el Estatuto General se agregan los siguientes:

I. Promover y difundir el desarrollo general de las humanidades, las ciencias y la tecnología... II. Conservar los valores culturales, difundiendo sus beneficios a toda la sociedad. III. Procurar la aplicación de los conocimientos humanísticos, científicos y tecnológicos en la solución de la problemática estatal y nacional (ver en siguiente columna punto IV)

V. Examinar teorías y difundir libremente el contenido de las diferentes doctrinas científicas y humanísticas. VI. Propiciar el mejoramiento y desarrollo cultural, moral y digno de los individuos y de la sociedad.

\begin{tabular}{|c|c|}
\hline Universidad & $\begin{array}{c}\text { Fines de la Universidad } \\
\text { Ley Orgánica de la UAEM: (22) Art. 3, de la Personalidad y Finalidad }\end{array}$ \\
\hline
\end{tabular}

Prestación de servicios públicos de educación de los tipos medio superior y superior, de investigación, de difusión de la cultura y extensión de los servicios. La finalidad de la Universidad es el

Universidad Autónoma del Estado de Morelos (UAEM) fortalecimiento y transformación de la sociedad a través de la ciencia, la educación y la cultura. En la consecución de esta finalidad la Institución tendrá como objetivo primordial insertarse eficiente y creativamente en su entorno, que no será sólo un campo de estudio sino,

fundamentalmente, objeto de transformación sobre el que se debe ejercer una permanente función crítica para la construcción de propuestas innovadoras y líneas de investigación encaminadas al desarrollo humano.
Principios o valores

Proyecto Académico 2014-2018. (21)

Equidad: ... búsqueda de la igualdad de oportunidades ... para competir, lo que exige acciones de apoyo diferenciado...

Transparencia: toda la información que se recopile y se procese será manejada a partir de criterios técnicos previamente establecidos, evitando que esto genere privilegios y oportunidades no previstas en las condiciones o normativas establecidas previamente, así como el manejo y acceso a los resultados.

Diversidad: se atenderá el respeto a las diferencias individuales, sin importar su

origen, raza, religión, etnias, posición social y orientación sexual, además se considerará... la orientación de las carreras que cada una de ellas imparten. Relevancia: se buscará el impacto de las funciones universitarias para el beneficio social.

Pertinencia: se atenderán las funciones sustantivas de la universidad en concordancia con los requerimientos de la sociedad.

Calidad: se trabajará en función a la necesidad de garantizar el progreso académico y administrativo en correspondencia con las exigencias que la calidad de la enseñanza superior requiere para el desarrollo social. Sustentabilidad y economía: se garantizará la eficiencia en el desarrollo de la gestión para cuantificar su impacto...

En el Estatuto general se habían consignado:

IV. Fomentar el respeto y la práctica de los valores y principios esenciales como la libertad, la justicia, democracia, solidaridad e igualdad.

$$
\text { Principios o valores }
$$

Plan de Desarrollo Institucional 2012-2018. (23)

\section{Valores que constituirán la esencia del Código Ético Institucional:}

Solidaridad: el reconocimiento de la alteridad, y la actitud de apoyo y colaboración con el otro...

Eticidad: el respeto a la autonomía y a la dignidad humanas...

Compromiso: con los seres humanos concretos, con la causa de su humanización a través de una educación que les permita aprender a conocer y hacer... aprender a ser a convivir y a preservar el medio ambiente ... a construir sentidos de vida y a definirse frente a los rumbos de la historia.

Honestidad: negación de prácticas deshonestas y estricto apego a los criterios, normas y principios éticos que definen la vida institucional...

Libertad: derecho para pensar, elegir y actuar de manera particular, con respeto a la diferencia y a los límites que nos impone la libertad de los otros.

Justicia: apego a los derechos y deberes de los universitarios... lucha por el acceso universal a la educación superior pública, por la autonomía y dignidad humanas, por la eliminación de las desigualdades sociales y por la consolidación de la democracia... Responsabilidad social: obligación de impulsar la educación media superior y superior pública y de avanzar hacia una visión incluyente del desarrollo nacional... Atributos de conducta distintivos de nuestra comunidad universitaria:

Autocrítica... Pluralidad ... Diálogo... Profesionalismo... Calidad... Coherencia... Lealtad. 
FUENTE: Elaboración propia. 


\section{Método}

Lo que aquí se reporta son resultados de tres equipos de investigación que participaron en la RIEICA para realizar una investigación en la modalidad de estudio colectivo de casos (24) (25) que incluyó dos fases. En la primera, cada equipo de investigación construyó su caso particular de estudio (análisis de la integridad académica en programas de posgrado seleccionados) y en la segunda se comparan los resultados obtenidos por los equipos de investigación que utilizaron las mismas estrategias metodológicas.

Para llevar a cabo esta comparación se revisaron los documentos institucionales de ambas universidades (ver supra). Además, se aplicó a docentes de los posgrados seleccionados un cuestionario con la finalidad de conocer cómo valoran las conductas de sus estudiantes que atentan contra la integridad académica, y qué hacen al respecto. instrumento base fue diseñado en 2006 por investigadores de la Universidad de Las Islas Baleares (26) y fue revisado y adaptado para ser aplicado en universidades mexicanas eliminando algunas preguntas, cambiando algunas palabras al uso común del español mexicano, y reorientando el sentido de las preguntas para que los docentes respondieran, ya que originalmente el cuestionario estaba dirigido a estudiantes para que ellos se autoevaluaran.

En el instrumento se exploran: el uso adecuado de fuentes de información, conductas deshonestas en el desarrollo de exámenes, plagio de trabajos (consultados en Internet o en fuentes impresas), falseamiento de referencias bibliográficas o datos. Incluye, además de preguntas abiertas y de opción múltiple, dos escalas tipo Likert, para señalar la gravedad de ciertas prácticas deshonestas, así como el grado de acuerdo o desacuerdo sobre la utilidad del uso de fuentes y la realización de trabajos académicos de parte de sus estudiantes. El análisis de las respuestas se orientó en dos direcciones: a) las diferencias encontradas por los docentes entre sus estudiantes de pregrado y posgrado; y b) las acciones que llevan a cabo para evitar que incurran en prácticas deshonestas.

\section{Participantes}

El cuestionario fue respondido por 28 profesores, con experiencia docente que va de dos a 28 años de servicio (67.9\% son mujeres), quienes actualmente imparten clases en dos posgrados de la UNACH y cuatro posgrados de la UAEM, así como en al menos una licenciatura de su unidad académica (ver Tabla 2). Es necesario anotar que estos profesores 
pueden impartir clases hasta en dos posgrados y en al menos una licenciatura de su unidad académica.

\begin{tabular}{|c|c|c|c|c|}
\hline \multicolumn{5}{|c|}{ TABLA 2. Profesores que respondieron el cuestionario en los casos UNACH y UAEM } \\
\hline CASO & $\begin{array}{c}\text { UNIDAD } \\
\text { ACADÉMICA }\end{array}$ & POSGRADO(S) & LICENCIATURAS & PROFESORES \\
\hline UNACH & $\begin{array}{l}\text { DES Ciencias } \\
\text { Sociales y } \\
\text { Humanidades de } \\
\text { la UNACH }\end{array}$ & $\begin{array}{l}\text { Maestría en Estudios } \\
\text { Culturales y/o Doctorado } \\
\text { en Estudios Regionales }\end{array}$ & Pedagogía & 10 \\
\hline \multirow{2}{*}{ UAEM } & $\begin{array}{l}\text { Instituto de } \\
\text { Ciencias de la } \\
\text { Educación } \\
\text { (Cuernavaca) de } \\
\text { la UAEM }\end{array}$ & $\begin{array}{l}\text { Maestría en Investigación } \\
\text { Educativa y Doctorado en } \\
\text { Educación }\end{array}$ & $\begin{array}{l}\text { Educación Física, Docencia, } \\
\text { Comunicación y Tecnología } \\
\text { Educativa, Enseñanza del } \\
\text { Inglés, Enseñanza del } \\
\text { Francés, Ciencias de la } \\
\text { Educación }\end{array}$ & 6 \\
\hline & $\begin{array}{l}\text { Facultad de } \\
\text { Estudios } \\
\text { Superiores de } \\
\text { Cuautla de la } \\
\text { UAEM }\end{array}$ & $\begin{array}{l}\text { Maestría y Doctorado en } \\
\text { Ciencias Sociales }\end{array}$ & $\begin{array}{l}\text { Sociología, Economía, } \\
\text { Relaciones Públicas, } \\
\text { Seguridad Ciudadana, } \\
\text { Psicología }\end{array}$ & 12 \\
\hline TOTAL & $\begin{array}{l}2 \text { Universidades, } \\
3 \text { Unidades } \\
\text { académicas }\end{array}$ & 7 programas de posgrado & 12 licenciaturas & 28 \\
\hline
\end{tabular}

Análisis de la información

La información recabada fue registrada en tablas estadísticas (para el caso de las preguntas de opción múltiple y escalas tipo Likert), así como capturadas y categorizada en el programa Atlas Ti para el caso de las preguntas abiertas. El análisis realizado fue mixto, primero comparando la frecuencia en la que los profesores perciben que los estudiantes de pregrado y posgrado incurren en prácticas deshonestas (análisis cuantitativo), para someter a examen el primer supuesto de la investigación y, posteriormente, indagando las acciones que los profesores implementan (análisis cualitativo) para conducir a sus estudiantes en el camino de la integridad académica.

\section{Prácticas que atentan contra la integridad académica}

Diferencias entre estudiantes de pregrado y posgrado

Con relación al supuesto de que los estudiantes de posgrado manifiestan un comportamiento ético superior a los de pregrado, en la Tabla 3 se presentan datos que lo corroboran pero que, en algunos casos, no muestran variaciones significativas. Destaca, por 
ejemplo, el hecho de que entre 10 y $14.3 \%$ de los estudiantes admite haber pagado a otro estudiante para que le haga su trabajo.

En los aspectos que se observa mayor grado de deshonestidad en la conducta de los estudiantes de pregrado, en comparación con los de posgrado, se encuentran los siguientes: entrega de trabajos realizados por otros o con fragmentos copiados de páginas web, copiar en exámenes, utilizando acordeones o dispositivos (como celulares, tablets o auriculares).

\begin{tabular}{|c|c|c|c|}
\hline \multicolumn{4}{|c|}{$\begin{array}{l}\text { TABLA 3. Conductas que atentan contra la integridad académica. } \\
\text { Diferencias entre estudiantes de pregrado y posgrado }\end{array}$} \\
\hline \multirow{2}{*}{ ASPECTO } & \multirow{2}{*}{ PREGRADO } & \multicolumn{2}{|c|}{ POSGRADO } \\
\hline & & MAESTRÍA & DOCTORADO \\
\hline Entrega de trabajos originales & 28.6 & 62.5 & 56.5 \\
\hline $\begin{array}{l}\text { Entrega de trabajos realizados previamente por otros } \\
\text { estudiantes }\end{array}$ & 70.3 & 8.7 & 9.1 \\
\hline $\begin{array}{l}\text { Entrega de trabajos copiando y pegando textos de } \\
\text { páginas web }\end{array}$ & 78.6 & 39.1 & 13.0 \\
\hline Entrega de trabajo descargado de internet sin cambios & 48.0 & 33.0 & 26.1 \\
\hline Pagar a otro estudiante para que haga el trabajo & 14.3 & 10.0 & 10.0 \\
\hline $\begin{array}{l}\text { Estudiantes que se ponen de acuerdo para copiarse } \\
\text { mutuamente en un examen }\end{array}$ & 42.0 & 8.0 & 13.0 \\
\hline $\begin{array}{l}\text { Utilización de dispositivos como celulares, tablets y } \\
\text { auriculares para copiar en exámenes }\end{array}$ & 53.0 & 30.0 & 16.0 \\
\hline $\begin{array}{l}\text { Uso de acordeón como estrategia para aprobar un } \\
\text { examen }\end{array}$ & 55.0 & 4.5 & 4.5 \\
\hline
\end{tabular}

En las respuestas de los profesores se observó que, si bien las conductas deshonestas son menos frecuentes en estudiantes de posgrado, siguen estando presentes, aunque sea pocas veces. Tal es el caso de los aspectos referidos en la Tabla 4 como son copiar fragmentos de fuentes impresas o de trabajos entregados previamente, incluir bibliografía no consultada y falsear datos.

\begin{tabular}{|c|c|c|c|c|}
\hline \multirow{3}{*}{ ASPECTO } & \multirow{3}{*}{ Diterencia entre } & \multirow{3}{*}{ PREGRADO } & \multirow{2}{*}{\multicolumn{2}{|c|}{ POSGRADO }} \\
\hline & & & & \\
\hline & & & MAESTRIA & DOCTORADO \\
\hline \multirow{2}{*}{$\begin{array}{l}\text { Copia de fragmentos de fuentes impresas } \\
\text { sin citar }\end{array}$} & Frecuente & 67.9 & 10.7 & \\
\hline & Pocas veces & 25.0 & 79.2 & 65.2 \\
\hline \multirow{2}{*}{$\begin{array}{l}\text { Copia de fragmentos de trabajos entregados } \\
\text { previamente }\end{array}$} & Frecuente & & & \\
\hline & Pocas veces & 50.0 & 33.0 & 40.0 \\
\hline \multirow{2}{*}{$\begin{array}{l}\text { Incluir en un trabajo bibliografía no } \\
\text { consultada }\end{array}$} & Frecuente & 40.0 & 37.0 & 13.0 \\
\hline & Pocas veces & 40.0 & 45.0 & 45.0 \\
\hline \multirow[t]{2}{*}{ Falsear datos en un trabajo académico } & Frecuente & 18.0 & 4.0 & 22.0 \\
\hline & Pocas veces & 40.0 & 39.0 & \\
\hline
\end{tabular}

Acerca de los conocimientos que, de acuerdo con los profesores poseen los estudiantes de pregrado y posgrado para realizar búsquedas de información, se observa (Tabla 5) que son 
superiores en los estudiantes de posgrado pero que sólo en algunos casos estos pueden ser considerados como excelentes.

\begin{tabular}{|c|c|c|c|c|}
\hline \multirow{2}{*}{ ASPECTO } & \multirow{2}{*}{ 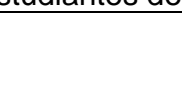 } & \multirow{2}{*}{ PREGRADO } & \multicolumn{2}{|c|}{ POSGRADO } \\
\hline & & & MAESTRÍA & DOCTORADO \\
\hline \multirow{4}{*}{$\begin{array}{l}\text { Conocimientos para buscar información } \\
\text { en bibliotecas con fines académicos }\end{array}$} & Nulos & 46.4 & 12.5 & 8.3 \\
\hline & Regulares & 39.4 & 50.0 & 45.8 \\
\hline & Buenos & 14.3 & 33.3 & 45.8 \\
\hline & Excelentes & & 4.2 & \\
\hline \multirow{4}{*}{$\begin{array}{l}\text { Conocimientos para buscar información } \\
\text { en internet con fines académicos }\end{array}$} & Nulos & 7.1 & & \\
\hline & Regulares & 64.3 & 33.3 & 29.2 \\
\hline & Buenos & 28.6 & 58.3 & 54.3 \\
\hline & Excelentes & & 8.3 & 16.6 \\
\hline
\end{tabular}

En cuanto a los indicadores que los docentes consideran que sus estudiantes toman en cuenta para discernir la fiabilidad de la información que encuentran en internet para realizar sus trabajos académicos, se observa que, conforme avanzan en el nivel educativo, prestan más atención a los autores, mientras que en pregrado predomina la facilidad para acceder a los materiales (ver Tabla 6).

\begin{tabular}{|l|r|r|r|}
\hline \multicolumn{3}{|c|}{ TABLA 6. Indicadores de fiabilidad tomados en cuenta por estudiantes de pregrado y posgrado para } \\
seleccionar información
\end{tabular}

También los docentes consideran que sus estudiantes de posgrado cuentan con competencias superiores al momento de seleccionar información (ver Tabla 7) y asumen con mayor responsabilidad la elaboración de sus trabajos académicos (ver Tabla 8).

\begin{tabular}{|l|r|r|r|}
\hline \multicolumn{1}{|c|}{ TABLA 7. Procedimiento seguido por estudiantes de pregrado y posgrado para seleccionar información } \\
\hline \multicolumn{1}{|c|}{ PROCEDIMIENTO } & PREGRADO & \multicolumn{2}{|c|}{ POSGRAD } \\
\cline { 3 - 4 } & 90.0 & 22.2 & MAESTRÍA \\
\hline $\begin{array}{l}\text { Utilizan los primeros 3-5 documentos que } \\
\text { aparecen en la pantalla }\end{array}$ & & 16.7 & 25.0 \\
\hline $\begin{array}{l}\text { Analizan los 50 primeros y escogen los que les } \\
\text { parecen más adecuados }\end{array}$ & & 61.1 & 37.5 \\
\hline $\begin{array}{l}\text { Escogen y analizan los que pertenecen a } \\
\text { organismos oficiales, revistas especializadas, } \\
\text { instituciones académicas }\end{array}$ & & & 18.8 \\
\hline $\begin{array}{l}\text { Realizan otras consultas mediante buscadores } \\
\text { diferentes y contrastan la información para } \\
\text { analizar los resultados obtenidos }\end{array}$ & & & \\
\hline Fuente: Elaboración propia a partir de respuestas de profesores. & & \\
\hline
\end{tabular}


Sin embargo, aún en los estudiantes de doctorado se presentan porcentajes significativos de conductas no éticas que impiden construir un posicionamiento crítico respecto de la información seleccionada, así como falta de responsabilidad frente a los trabajos encomendados por los profesores.

\begin{tabular}{|l|r|r|r|}
\hline \multicolumn{3}{|c|}{ TABLA 8. Indicadores de fiabilidad tomados en cuenta por estudiantes de pregrado y posgrado para } \\
seleccionar información
\end{tabular}

Acciones docentes para fortalecer la integridad académica

Tomando en cuenta que, aunque con algunas diferencias porcentuales, tanto en pregrado como en posgrado los estudiantes cometen faltas que atentan contra la integridad académica, es de esperarse, y este es el segundo supuesto que guio este estudio, que los docentes tengan que actuar de alguna forma y que, al no contar con un documento institucional sobre la conducta ética esperada, sus acciones (sea para imponer sanciones, reflexionar con sus estudiantes o proponer estrategias de enseñanza, entre otras posibilidades), se encuentren desarticuladas y asuman formas muy diversas que no permiten conducir el proceso de formación en un sentido claro.

Al preguntarle a los profesores cuáles son los pasos que piden habitualmente a sus alumnos de pregrado y posgrado para que realicen búsquedas de información y escriban un trabajo académico, encontramos que no establecen diferencias. Asimismo, existe la idea generalizada entre ellos de que en muchos casos los estudiantes incurren en estas prácticas porque otros docentes no les han enseñado o no les han corregido.

Con la información recabada en las preguntas abiertas del cuestionario aplicado a los profesores se hizo una codificación axial y se extrajeron seis categorías que se describen a continuación: 
Enseñanzas: centradas en un conjunto de orientaciones que permiten a los estudiantes realizar un trabajo. Entre estas destacan aquellas relacionadas con el acceso a material que puede ser consultado en internet, por ejemplo: bases de datos (como Redalyc o google académico); localizar las fuentes de información, eligiendo un período de búsqueda (información actualizada); realizar búsquedas (de material bibliográfico e informes estadísticos) y diferenciar fuentes de información con carácter científico (información confiable que pueda respaldar sus trabajos). Esta última aplica también a la realización de trabajos tomando como base la búsqueda de material impreso en bibliotecas de la universidad o externas.

Una preocupación central de los docentes en este rubro es acerca del uso del aparato crítico en los trabajos, lo que incluye: cómo citar textualmente y parafrasear (evitando copias innecesarias y no referenciadas), la anotación de las referencias documentales y enseñanzas relacionadas con cómo utilizar la información para redactar comparando ideas originales con las de los autores. Un docente comentó que era importante promover la discusión en equipos y realizar trabajo colaborativo.

Medidas preventivas: incluyen las expectativas del profesor para que el estudiante se conduzca con integridad académica. En este rubro los docentes proponen anexar normas detalladas de cómo citar (APA o ISSO) y proponer materiales de consulta; pedir que reflexionen lo qué quieren decir y cómo decirlo; recomendar que seleccionen las lecturas más relacionadas con el tema, revisen los autores, las lean varias veces y realicen resúmenes. Se considera también la necesidad de explicar los aspectos que se van a tomar en cuenta para la evaluación, incluyendo las reglas de uso del aparato crítico; en el caso de los exámenes recomendar que sean honestos y en la redacción de las tesis recordar que las ideas a desarrollar deben ser originales.

Revisiones: referidas al seguimiento y corrección que hacen los profesores de los avances de sus estudiantes. En esta categoría admiten que es necesario leer los trabajos puntualmente, hacerles observaciones por escrito sobre el documento y comentar con ellos las fallas en las que incurren. Una actividad referida recurrentemente por los docentes es la búsqueda en Internet de posibles plagios (para lo cual utilizan frases o párrafos sospechosos).

Medidas correctivas: colocadas en el ámbito "privado" de su clase conminando al estudiante a comprender lo cuestionable o grave de la conducta, dándole oportunidad de corregir. Incluye hablar con los estudiantes explicando la gravedad de la falta (trabajos escritos o exámenes), 
motivándolos a reflexionar sobre su actuar y las posibles consecuencias (castigos o sanciones). Entre las medidas correctivas se insiste en las formas correctas de citación y se brinda otra oportunidad para que entreguen el trabajo corregido.

Sanciones: medidas que toman los profesores por su cuenta o aludiendo a alguna normativa de orden general. Estas medidas consisten en bajar puntos en la calificación si la falta no es muy grave o invalidar el examen o el trabajo si la falta es grave; exigir que repitan el trabajo con sus propios conceptos y/o dar un trabajo extra; en caso de reincidencia, denunciar ante la Comisión Académica y aplicar la normativa al respecto (suspensión o baja).

Control: lineamientos que establecen los profesores exigiendo el cumplimiento a sus estudiantes, especificando o "recomendando" las fuentes que se deben consultar y cómo entregar los trabajos. En este caso se llegan a hacer señalamientos estrictos sobre qué fuentes considerarán válidas, cómo y dónde debe hacerse la búsqueda de información.

Es claro que los docentes comparten algunas estrategias de enseñanza, así como algunas formas para responder ante la deshonestidad académica, sin embargo, sus actuaciones reflejan un trabajo aislado.

\section{Conclusiones}

De acuerdo con el objetivo planteado se observa que las prácticas que atentan contra la integridad académica efectivamente disminuyen conforme aumenta el grado de estudios, pero siguen estando presentes, lo que lleva a los docentes a cuestionarse como actuar.

Los resultados obtenidos en este estudio, los docentes son conscientes que, tanto en pregrado como en posgrado debe trabajarse de manera permanente y consistente en dirección a la consecución de la integridad académica. No obstante, para avanzar en ese sentido es necesario hacerlo a través de un trabajo colaborativo que integre a los docentes, en primera instancia, pues es importante que dialoguen entre ellos acerca de las estrategias que cada uno impulsa en su quehacer académico. Se requiere discutir y llegar a puntos de encuentro acerca del concepto de integridad académica institucional al que se aspira.

Es importante generar el consenso entre la academia en el sentido de anteponer la información y la formación, a la persecución y sanción de las actitudes deshonestas; lo primero abona a la creación de una cultura académica ética, alineada con las políticas de las instituciones, que permita transitar de la normalización del fraude a la normalización de la integridad. Para ello es necesario que este diálogo se amplíe para incluir a las autoridades 
universitarias y los estudiantes, incidiendo en la construcción de una ética institucional. La honestidad es un valor fundamental de la integridad académica, en tanto refleja un "comportamiento universitario auténtico, coherente con valores de verdad y justicia que se traducen en transparencia institucional como rasgo distintivo". (27)

Sin la participación decidida, comprometida y responsable de la comunidad académica a través de una reflexión y una deliberación dialogada, sentar las bases de esa integridad académica se torna mucho más complicado. Las autoridades deben promover y sensibilizar a su comunidad académica a través de información de los principios y valores institucionales, para coadyuvar a la formación de un clima organizacional ético y responsable en el que los estudiantes, docentes, investigadores y autoridades encuentren el espacio idóneo para confrontar sus posturas y reflexiones de una manera abierta y crítica.

Independientemente de las razones por las cuales un profesor se encuentra laborando en una IES, es necesario que asuma un compromiso honesto. Una manera, aunque no la única, de enseñar es a través del ejemplo, los profesores que imparten clases en pregrado y posgrado deben ser congruentes entre lo que dicen y lo que hacen, esto sin duda coadyuvará a lograr que los futuros profesionales asuman un compromiso ético. Hay que tener presente que algunos estudiantes asumen prácticas incorrectas o aparentemente deshonestas por falta de información y formación. La integridad académica es una tarea que requiere de una construcción gradual, pausada y formativa; no se trata de un fin en sí misma, sino de un medio de amplias repercusiones y en ese proceso, el profesor puede resultar un excelente acompañante.

En el caso de los estudiantes que reciben una subvención económica mensual para dedicarse a sus estudios, como es el caso de los posgrados reconocidos en el PNPC del CONACYT, las conductas deshonestas cuestionan también las políticas institucionales, la política científica nacional y el uso de los recursos destinados a la formación de investigadores.

Si bien este estudio aporta información de dos casos, es necesario continuar y profundizar la investigación sobre estas prácticas académicas deshonestas para erradicarlas, abarcado distintas etapas educativas y tomando en cuenta diferentes actores, dado que la problemática que aquí se abordas rebasa las fronteras institucionales y nacionales.

\section{Notas}

(*) Las autoras forman parte de la Red de Investigadores para el Estudio de la Integridad y Calidad Académica (RIEICA) y en este artículo exponen resultados del proyecto de investigación "Evaluación de la Calidad del Profesorado en Universidades de México y España", inscrito por esta red en la Convocatoria 2015 de Apoyo a la integración de redes temáticas de colaboración 
académica emitida por el Programa para el Desarrollo Profesional Docente, para el Tipo Superior (clave PRODEP/DSA/103.5/15/11032 /28-09- 2015).

(1) Stake, Robert E. Investigación con estudio de casos, Madrid, Morata, 1999.

(2) Comas, Rubén (et al). "La integridad académica entre el alumnado universitario español", en Estudios Pedagógicos, (Valdivia), vol. XXXVII, núm. 1, 2011, pp. 207-225. Recuperado de (6/05/2017): https://dialnet.unirioja.es/servlet/articulo?codigo=4881559

(3) Bretag, Tracey. "Challenges in Addressing Plagiarism in Education", en PLoS Med 10(12): e1001574, 2013, doi:10.1371/journal.pmed.1001574

(4) Vasconcelos, Sonia. "La integridad y la conducta responsable en la investigación científica: los grandes desafíos", en Revista pesquisa, Brasil, Editorial 200, 2012, (s/p). Recuperado de (23/04/ 2017): http://revistapesquisa.fapesp.br/es/2012/10/23/la-integridad-y-la-conducta-responsable-enla-investigaci\%C3\%B3n-cient\%C3\%ADfica-los-grandes-desaf\%C3\%ADos/

(5) Comas (et al.), Op. Cit., pp. 208-210.

(6) Ibid, p. 201.

(7) RIEICA. Evaluación de la calidad del profesorado en Universidades de México y España, México, PRODEP, 2015. Documento de trabajo.

(8) Comas (et al.), Op. Cit., p. 207.

(9) Guerrero, Sánchez Pablo, Mercado Yebra, Joaquín, e Ibarra Uribe, Luz Marina. "La deshonestidad, elemento que altera la integridad en las prácticas académicas en las Instituciones de Educación Superior" en Revista Investigación y formación pedagógica, número 5(3), Venezuela, CIEGC, 2017, pp.6-25. Recuperado de (9/05/2017):

http://revistas.upel.edu.ve/index.php/revinvformpedag/article/view/5183

(10) Pineda, Gómez José Alfredo, Albarrán Millán, Delia y Muñoz, Martínez Aurora del Socorro. "El plagio académico y la integridad en los alumnos del posgrado de la Universidad Autónoma de Guerrero", en Devenir, No. 31, México, UNAH, 2016, pp. 171-182.

(11) Lara Piña, Fernando y Pons Bonals, Leticia. "Formación de investigadores y responsabilidad social. Competencias de estudiantes de nivel posgrado", en Ibáñez-Martín, J. y Fuentes, J. (editores), Actas Del VIII Congreso Internacional de Filosofía de la Educación, España, Universidad Católica de Valencia San Vicente Mártir, 2017.

(12) ICAI. "Fundamental Values Project" en International Center for Academic Integrity (2017). Recuperado de (14/05/2017): http://www.academicintegrity.org/icai/resources-2.php

(13) UDEM. Sistema de integridad de la UDEM. Monterrey, Nuevo León, Universidad de Monterrey. 2017. Recuperado de (10/05/2017): http://www.udem.edu.mx/Esp/Somos-UDEM/Pages/Sistemade-integridad.aspx

(14) Teh, J.; Willis, T y Rouf, M., O. A. (2014). Quotes about Academic Integrity from American University in Dubai (AUD) Undergraduate Students. Recuperado de:

http://www.academicintegrity.org/icai/assets/AUD_Integrity_Quotes.pdf.

(15) Ruipérez, Germán; García-Cabrero, José-Carlos. "Plagio e integridad académica en Alemania" Comunicar, vol. XXIV, núm. 48, España, Grupo Comunicar Huelva, 2016, pp. 16. Recuperado de (11/04/2017): http://www.redalyc.org/articulo.oa?id=15846325001 
(16) Ibid., p. 16.

(17) Universidad de Jaén. "Plagio y honestidad académica". Universidad de Jaén, 2017. Recuperado de (14/05/2017):

http://blogs.ujaen.es/abiertobuja/?page id=1201iversidad\%20de\%20Ja\%C3\%A9n,\%202017

(18) Universidad de Jaén. "Principios fundamentales de la integridad académica". Universidad de Jaén, 2015. Recuperado de: http://blogs.ujaen.es/abiertobuja/wpcontent/uploads/2015/03/principios-integridad-icai.pdf

(19) UNACH. Ley Orgánica de la Universidad Autónoma de Chiapas, Tuxtla Gutiérrez, Chiapas, Universidad Autónoma de Chiapas, 1989.

(20) UNACH. Estatuto General de la Universidad Autónoma de Chiapas, Tuxtla Gutiérrez, Chiapas, Universidad Autónoma de Chiapas, 1995.

(21) UNACH. Proyecto Académico 2014-2018 de la Universidad Autónoma de Chiapas, Tuxtla Gutiérrez, Chiapas, Universidad Autónoma de Chiapas, 2015.

(22) UAEM. Ley Orgánica de la Universidad Autónoma del Estado de Morelos, Cuernavaca, Morelos, Universidad Autónoma del Estado de Morelos, 2008.

(23) UAEM. Plan Institucional de Desarrollo (PIDE), Morelos, Universidad Autónoma del Estado de Morelos, Cuernavaca, Morelos, Universidad Autónoma del Estado de Morelos, 2012.

(24) Stake, R. Op cit.

(25) Stake, R. "Estudios de casos cualitativos", en N. K. Denzin e Y. S. Lincoln (comps), Estrategias de investigación cualitativa, Barcelona, Gedisa, 2013.

(26) Comas (et al.), Op. Cit., p. 207.

(27) UAEM. Plan Institucional de Desarrollo (PIDE), Cuernavaca, Morelos: Universidad Autónoma del estado de Morelos, 2012, p. 17.

Recibido: julio de 2017.

Aprobado: octubre de 2017.

\section{Para citar este trabajo}

Escalante Ferrer, A. E.; Ibarra Uribe, L. M.; Pons Bonals, L. "Docentes de dos universidades públicas mexicanas ante la integridad académica de sus estudiantes" en Cuadernos de H Ideas [En línea], vol. 11, № 11, diciembre 2017, consultado...; URL: http://perio.unlp.edu.ar/ojs/index.php/cps/article/view/4262 